International Journal of Pure and Applied Mathematics

Volume 86 No. 5 2013, 859-869

ISSN: 1311-8080 (printed version); ISSN: 1314-3395 (on-line version)

url: http://www.ijpam.eu

doi: http://dx.doi.org/10.12732/ijpam.v86i5.9

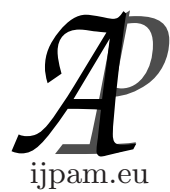

\title{
QUALITATIVE PROPERTIES OF SELF-SIMILAR SOLUTIONS OF A NONHOMOGENEOUS EQUATION
}

\author{
Badia Hamri ${ }^{1}$, Abdelilah Gmira ${ }^{2}$, Youssef Jabri ${ }^{3} \S$ \\ ${ }^{1,2}$ Department of Mathematics \\ Faculty of Science \\ University Abdelmalek Essaâdi \\ B.P. 2121, Tétouan, MOROCCO \\ ${ }^{3}$ Department of Mathematics \\ National School of Applied Sciences \\ University Mohammed I \\ B.P. 669 , Oujda, MOROCCO
}

Abstract: In [2], we have proved the existence and uniqueness of self-similar radially symmetric solutions for the nonhomogeneous equation

$$
u_{t}=\operatorname{div}\left(|\nabla u|^{p-2} \nabla u\right)+|x|^{l}|u|^{q-1} u, \quad(t, x) \in(0,+\infty) \times \mathbb{R}^{N} .
$$

where $p>2, q \geq 1, N \geq 1,-p<l,-N<l$.

We have studied the asymptotic behaviour of such a solution in [3]. Our aim, in this paper, is to classify the solutions (positiveness, compact support,...) according to the parameters $p, q$ and $l$.

AMS Subject Classification: 35K55, 35K65

Key Words: qualitative properties, self-similar solution, parabolic problem, nonhomogeneous equation

\section{Introduction}

In [2], we have investigated the existence and uniqueness of self-similar radially

Received: May 15, 2013

(C) 2013 Academic Publications, Ltd.

$\S$ Correspondence author url: www.acadpubl.eu 
symmetric solutions of the parabolic nonhomogeneous equation

$$
w_{t}=\Delta_{p} w+|x|^{l}|w|^{q-1} w, \quad \text { in } \mathbb{R}^{\mathbb{N}} \times(0,+\infty)
$$

where $p>2, q \geq 1, N \geq 1,-p<l<0,-N<l<0$.

Self-similar solutions have the form

$$
w(x, t)=t^{-\alpha} U\left(t^{-\beta} x\right), \text { in } \mathbb{R}^{\mathbb{N}} \times(0,+\infty),
$$

And the profile $U$ satisfies the nonhomogeneous elliptic equation

$$
\Delta_{p} U+\beta x \nabla U+\alpha U+|x|^{l}|U|^{q-1} U=0, \text { for } x \in \mathbb{R}^{\mathbb{N}}
$$

where the scaling factors are given by

$$
\alpha=\frac{p+l}{p(q-1)+l(p-2)}, \beta=\frac{q-p+1}{p(q-1)+l(p-2)} \text { and } l \neq-\frac{p(q-1)}{p-2}
$$

Radially symmetric solutions satisfy

$$
U(y)=u(|y|)=u(r) .
$$

In [3], we studied the asymptotic behaviour of the solution when $\alpha$ and $\beta$ are both positive.

In this paper, we shall consider the qualitative properties of the solutions of $(P)$ like positiveness, and compact support according to the values of the parameters $\alpha, \beta, p, q$ and $l$.

\section{Qualitative Properties of the Solutions}

We first start with the following result

Proposition 2.1. Assume $p>2, \alpha \geq N \beta>0$. There is no positive solution of $(P)$.

Proof. Suppose by contrary that $u$ is a positive solution of problem $(P)$. Therefore after integration from 0 to $r$, we get

$$
\begin{aligned}
r^{N-1}\left|u^{\prime}\right|^{p-2} u^{\prime}=-\beta r^{N} u(r)-(\alpha-N \beta) \int_{0}^{r} s^{N-1} u(s) d s & \\
& -\int_{0}^{r} s^{l+N-1} u^{q}(s) d s
\end{aligned}
$$


As $\alpha \geq N \beta>0$, the following inequality holds

$$
\left|u^{\prime}\right|^{p-2} u^{\prime}<-\beta r u(r) \text { for all } r \in \mathbb{R}
$$

Hence

$$
u^{\frac{p-2}{p-1}}<a^{\frac{p-2}{p-1}}-\frac{p-2}{p} \beta^{\frac{1}{p-1}} r^{\frac{p}{p-1}}
$$

The following result ensures the existence of strictly positive solutions when $0<\alpha<N \beta$

Proposition 2.2. Assume $p>2,0<\alpha<N \beta,-\frac{p}{p-1}<l<0$ and $-N<l$. Then if $q>1-\frac{l(p-2)}{p}$ (resp. $q<1-\frac{l(p-2)}{p}$ ) there exists $a_{*}$ such that if $a \in\left(0, a_{*}\right)$ (resp. $a \in\left(a_{*}, \infty\right)$ ), the solution of $(P)$ is strictly positive.

To prove this result we write $(P)$ as a perturbation of the following problem

$$
(Q)\left\{\begin{array}{l}
\left(\left|w^{\prime}\right|^{p-2} w^{\prime}\right)^{\prime}+\frac{N-1}{r}\left|w^{\prime}\right|^{p-2} w^{\prime}+\alpha w+\beta r w^{\prime}=0 \\
w(0)=1, \lim _{r \rightarrow 0} r^{N-1}\left|w^{\prime}\right|^{p-2} w^{\prime}=0 .
\end{array}\right.
$$

For this purpose, let $a$ be a real positive and define the function $v$ by

$$
u(r)=a v(\xi), \xi=a^{-\frac{p-2}{p}} r \text { for any } r>0 .
$$

where $u$ is the solution of $(P)$. Then $v$ satisfies

$$
\left(Q_{b}\right)\left\{\begin{array}{l}
\left(\left|v^{\prime}\right|^{p-2} v^{\prime}\right)^{\prime}+\frac{N-1}{\xi}\left|v^{\prime}\right|^{p-2} v^{\prime}+\alpha v+\beta \xi v^{\prime}+b \xi^{l}|v|^{q-1} v=0 \\
v(0)=1, \lim _{r \rightarrow 0} \xi^{N-1}\left|v^{\prime}\right|^{p-2} v^{\prime}=0 .
\end{array}\right.
$$

where $b=a^{q-1+\frac{l(p-2)}{p}}$.

To emphasize the dependence of $v$ to the parameter $b$, we denote it by $v(\zeta, b)$. If we set $w$ the solution of $(Q)$ the following result holds

Proposition 2.3. Assume $p>2,0<\alpha<N \beta,-\frac{p}{p-1}<l<0$ and $-N<l$. For every $0<\varepsilon<\frac{\alpha}{2 \beta N}$ and $R>0$ there exists $b_{0}$ such that for $b \in\left(0, b_{0}\right)$ we have

$$
|v(\zeta, b)-w(\zeta)| \leq \varepsilon \quad \text { in }[0, R] .
$$

Proof. First, we claim that $w$ is strictly positive and strictly decreasing. If not, let $\xi_{0}$ the first zero of $w$. Then integrate $(2.3)$ on $\left(0, \xi_{0}\right)$ we get

$$
\xi_{0}^{N-1}\left|w^{\prime}\right|^{p-2} w^{\prime}\left(\xi_{0}\right)=-(\alpha-N \beta) \int_{0}^{r_{0}} s^{N-1} w(s) d s .
$$


Now $0 \leq \alpha<N \beta$, the right hand side is strictly positive as the left hand side is negative which is a contradiction. Consequently $0<w(\xi) \leq 1$ and $\lim _{\xi \rightarrow+\infty} w(\xi)=0$. The rest of the proof will devided into two steps

Step 1. $|v(\xi, b)-w(\xi)| \leq \varepsilon$ in $\left[0, R_{0}\right]$, where $R_{0}=w^{-1}\left(1-\frac{\alpha}{2 \beta N}\right)$

Take $b$ small enough. As $v$ and $w$ are continuous fonctions and satisfying $v(0, b)=w(0)$, then there exists $R(b)$ such that for any $\xi \in[0, R(b)]$

$$
|v(\xi, b)-w(\xi)| \leq \varepsilon
$$

If $R(b) \geqslant R_{0}$, we have the desired result.

Assume in the sequel $R_{0}>R(b)$. As $w$ is decreasing and satisfies $0<w(\xi) \leq$ $w(\xi)=1$; then without loss of generality we can assume that

$$
\varepsilon \leq w\left(R_{0}\right)<w(R(b))<w(\xi) \leq 1 \text { in }[0, R(b)]
$$

Then (2.8) implies that for any $\xi \in[0, R(b)]$

$$
0<w\left(R_{0}\right)-\varepsilon<w(\xi)-\varepsilon<v(\xi, b)<\varepsilon+w(\xi)
$$

Now recall that $w$ and $v$ are given by the following implicit formula

$$
w(\xi)=1-\int_{0}^{\xi}|f(s)|^{\frac{2-p}{p-1}} f(s) d s \quad \text { for } \xi \in[0, R(b)]
$$

and

$$
v(\xi, b)=1-\int_{0}^{\xi}|g(s)|^{\frac{2-p}{p-1}} g(s) d s \quad \text { for } \xi \in[0, R(b)]
$$

where

$$
f(s)=\beta s w(s)+(\alpha-N \beta) s^{1-N} \int_{0}^{s} \sigma^{N-1} w(\sigma) d \sigma
$$

and

$$
\begin{aligned}
& g(s)=\beta s v(s, b) \\
& \quad+(\alpha-N \beta) s^{1-N} \int_{0}^{s} \sigma^{N-1} v(\sigma, b) d \sigma+b s^{1-N} \int_{0}^{s} \sigma^{l+N-1} v^{q}(\sigma, b) d \sigma
\end{aligned}
$$

From the choice of $R_{0}$, we get

$$
w(\xi) \geqslant w\left(R_{0}\right) \geqslant 1-\frac{\alpha}{2 \beta N} \text { for any } \xi \in[0, R(b)] .
$$


and consequently $f$ satifsfies

$$
f(s) \geqslant\left[\beta w\left(R_{0}\right)+\frac{\alpha}{N}-\beta\right] s \text { for any } s \in[0, R(b)],
$$

On the other hand, combining (2.10), (2.14) and (2.15) and since $0<v \leq 1$ in $[0, R(b)]$, we get

$$
g(s) \geqslant C\left(R_{0}\right) s \text { for any } s \in[0, R(b)],
$$

where

$$
C\left(R_{0}\right)=\beta w\left(R_{0}\right)+\frac{\alpha}{N}-\beta-\beta \varepsilon+\frac{b_{0}}{N+l} R_{0}^{l}\left(w\left(R_{0}\right)-\varepsilon\right)^{q} .
$$

Now choose $b$ such that

$$
b \leq \beta \varepsilon(N+l) R_{0}^{-l}\left(w\left(R_{0}\right)-\varepsilon\right)^{-q} .
$$

So

$$
C\left(R_{0}\right)<\beta w\left(R_{0}\right)+\frac{\alpha}{N}-\beta
$$

Consequently if we let

$$
h(s)=\inf (|f(s)|,|g(s)|) \quad s \in[0, R(b)]
$$

we get from (2.11)-(2.12) and (2.18) the following inequality

$$
|v(\xi, b)-w(\xi)| \leq c^{\frac{2-p}{p-1}}\left(R_{0}\right) \int_{0}^{\xi}|f(s)-g(s)| s^{\frac{2-p}{p-1}} d s
$$

for any $\xi \in[0, R(b)]$.

Now using (2.13) and (2.14) we get

$$
\begin{aligned}
& \int_{0}^{\xi}|f(s)-g(s)| s^{\frac{2-p}{p-1}} d s \leq+\beta \int_{0}^{\xi} s^{\frac{1}{p-1}}\left|v\left(s, b_{0}\right)-w(s)\right| d s \\
& \quad+|\alpha-N \beta| \int_{0}^{\xi} s^{\frac{1}{p-1}-N} \int_{0}^{s} \sigma^{N-1}\left|v\left(\sigma, b_{0}\right)-w(s)\right| d \sigma d s \\
& \quad+b \int_{0}^{\xi} s^{\frac{1}{p-1}-N} \int_{0}^{s} \sigma^{N+l-1} v^{q}(\sigma, b) d \sigma .
\end{aligned}
$$

Using again $0<v \leq 1$, the following estimate holds in $[0, R(b)]$.

$$
\left|v\left(\xi, b_{0}\right)-w(\xi)\right| \leq K b c^{\frac{2-p}{p-1}}\left(R_{0}\right) R_{0}^{l+\frac{p}{p-1}}
$$




$$
+K c^{\frac{2-p}{p-1}}\left(R_{0}\right) \int_{0}^{\xi} s^{\frac{1}{p-1}}|v(s, b)-w(s)| d s
$$

where we denote by $K$ different constants.

Applying now Gronwall's inequality, we get

$$
|v(\xi, b)-w(\xi)| \leq b K c^{\frac{2-p}{p-1}}\left(R_{0}\right) R_{0}^{l+\frac{p}{p-1}} e^{K c^{\frac{2-p}{p-1}}\left(R_{0}\right)} \quad \text { in }[0, R(b)] .
$$

It follows that by choosing $b$ small enough so that the right hand side of $(2.24)$ is less than $\varepsilon$, we can take $R(b)=R_{0}$.

Step 2. The following estimate holds

$$
|v(\xi, b)-w(\xi)| \leq \varepsilon \text { for any } \xi \in\left[R_{0}, R\right]
$$

As to prove the global existence, we introduce the energy function associated to problem $\left(Q_{b}\right)$

$$
E_{b}(\xi)=\frac{p}{p-1}\left|v^{\prime}\right|^{p}+\frac{\alpha}{2} v^{2}+b \xi^{l} \frac{|v|^{q+1}}{q+1}
$$

It is easy to see that $E_{b}$ is decreasing in $\left[R_{0}, R\right]$. So, the following inequality holds

$$
\frac{\alpha}{2} v^{2}(\xi, b) \leq E_{b}\left(R_{0}\right)=\frac{p-1}{p}\left|v^{\prime}\left(R_{0}, b\right)\right|^{p}+\frac{\alpha}{2} v^{2}\left(R_{0}, b\right)+R_{0}^{l} \frac{\left|v\left(R_{0}, b\right)\right|^{q+1}}{q+1}
$$

for any $\xi \in\left[R_{0}, R\right]$ and any $b \in[0, b]$.

First, Step 1 implies that $v(., b)$ is bounded in $\left[0, R_{0}\right]$ for any $b \in\left[0, b_{0}\right]$ (with small $\left.b_{0}\right)$. On the hand, integrating $(2.5)$, we obtain

$$
\begin{aligned}
\left|v^{\prime}\left(R_{0}, b\right)\right|^{p-1} \leq|\alpha-N \beta| R_{0}^{1-N} & \int_{0}^{R_{0}} s^{N-1}|v(s, b)| d s \\
& +R_{0}^{l} \int_{0}^{R_{0}}|v|^{q}(s, b) d s+\beta\left|R_{0}\right| v\left(R_{0}, b\right) .
\end{aligned}
$$

and then $v^{\prime}\left(R_{0}, b\right)$ is bounded. Thereby (2.27) gives the boundedeness in $\left[R_{0}, R\right]$ of $v(\xi, b)$. Finally integrating equation $(2.5)$ we deduce that $v^{\prime}(\xi, b)$ is uniformly bounded in $\left[R_{0}, R\right]$ for any $b \in\left[0, b_{0}\right]$. By using Arzela-Ascoli theorem, there exists a function $\tilde{w}:\left[R_{0}, R\right] \rightarrow \mathbb{R}^{+}$such that $v(., b)$ converges uniformly in $C^{1, \alpha}\left(\left[R_{0}, R\right]\right)$ to $\tilde{w}$ as $b$ goes to 0 . Hence, if we integrate equation (2.5) on $\left[R_{0}, \xi\right]$ (where $0<R_{0}<\xi \leq R$ ) and letting $b$ go to 0 , we obtain 


$$
\begin{array}{r}
\xi^{N-1}\left|\widetilde{w}^{\prime}\right|^{p-2} \widetilde{w}^{\prime}(\xi)-R_{0}^{N-1}\left|\widetilde{w}^{\prime}\right|^{p-2} \widetilde{w}^{\prime}\left(R_{0}\right)=-\beta \xi^{N} \widetilde{w}(\xi)+\beta R_{0}^{N} \widetilde{w}\left(R_{0}\right) \\
-(\alpha-N \beta) \xi^{1-N} \int_{R_{0}}^{\xi} s^{N-1} \widetilde{w}(s) d s .
\end{array}
$$

Moreover, by letting $b$ go to 0 in the equation:

$$
\begin{aligned}
R_{0}^{N-1}\left|v^{\prime}\right|^{p-2} v^{\prime}\left(R_{0}\right)= & -\beta R_{0}^{N} v\left(R_{0}\right)-(\alpha-N \beta) \int_{0}^{R_{0}} s^{N-1} v(s) d s \\
& -b \int_{0}^{R_{0}} s^{N+l-1}|v|^{q-1} v(s) d s
\end{aligned}
$$

and using step1, we obtain

$$
R_{0}^{N-1}\left|\widetilde{w}^{\prime}\right|^{p-2} \widetilde{w}^{\prime}\left(R_{0}\right)=-\beta R_{0}^{N} w\left(R_{0}\right)-(\alpha-N \beta) \int_{0}^{R_{0}} s^{N-1} w(s) d s
$$

Recall (2.3), we deduce $\widetilde{w}^{\prime}\left(R_{0}\right)=w^{\prime}\left(R_{0}\right)$. Consequently (2.29) implies that $\widetilde{w}(\xi)=w(\xi)$ for any $\xi \in\left[R_{0}, R\right]$, and thereby (2.25) follows.

Combining step1 and step2, we get (2.6), which completes the proof.

Now Proposition 1.2, is just a consequence of Proposition 1.3.

In the sequel we introduce the following transformation

$$
u(r)=a v(\xi), \quad \xi=a^{\frac{q+1-p}{p+l}} r
$$

then $v$ satisfies

$$
\left(S_{b}\right)\left\{\begin{array}{l}
\left(\left|v^{\prime}\right|^{p-2} v^{\prime}\right)+\frac{N-1}{\xi}\left|v^{\prime}\right|^{p-2} v^{\prime}+\xi^{l}|v|^{q-1} \xi+b\left(\alpha v+\beta \xi v^{\prime}\right)=0 \\
v(0)=1, \quad \lim _{\xi \rightarrow 0} \xi^{N-1}\left|v^{\prime}\right|^{p-2} v^{\prime}(\xi)=0
\end{array}\right.
$$

where $b=a^{\gamma}$ with $\gamma=-\frac{l(p-2)+p(q-1)}{p+l}$.

Let $w$ the solution following problem

$$
(S)\left\{\begin{array}{l}
\left(\left|w^{\prime}\right|^{p-2} w^{\prime}\right)^{\prime}+\frac{N-1}{\xi}\left|w^{\prime}\right|^{p-2} w^{\prime}+\xi^{l}|w|^{q-1} w=0 \\
w(0)=1, \quad \lim _{\xi \rightarrow 0} \xi^{N-1}\left|w^{\prime}\right|^{p-2} w^{\prime}(\xi)=0
\end{array}\right.
$$

Using [3], $w$ is unique and nonpositive decreasing solution. Then there exists $0<R_{0}<R_{*}$ such that

$$
\left\{\begin{array}{l}
w(r)>0 \text { for } r \in\left[0, R_{0}[\right. \\
w\left(R_{0}\right)=0 \text { and } w(r)<0 \text { for } r \in\left[R_{0}, R_{*}\right]
\end{array}\right.
$$

Note that $\left(S_{b}\right)$ is an approximate of $(S)$ when $b$ goes to 0 . In fact the following result holds 
Proposition 2.4. Assume $p>2,0<\alpha<N \beta,-\frac{p}{p-1}<l<0,-N<l$ and $q \neq 1-\frac{l(p-2)}{p}$ such that $q<\frac{p(l+N)}{N-p}-1$. Let $R_{*}$ given in $(2.35)$, then for every small positive $\varepsilon$ there exists an $b_{*}$ such that for any $b \in\left(0, b_{*}\right)$ we have

$$
|v(\xi, b)-w(\xi, b)| \leq \xi \quad \text { in }\left[0, R_{*}\right]
$$

The argument to show this result is essentially the same as in the previous proposition.

As a consequence, we get

Corollary 2.1. Assume $p>2, q<\frac{p(l+N)}{N-p}-1,0<\alpha<N \beta,-\frac{p}{p-1}<l<0$ and $-N<l$. Then if $q>1-\frac{l(p-2)}{p}$ ( resp. $q<1-\frac{l(p-2)}{p}$ ) there exists $a^{*}$ such that if $a \in\left(0, a^{*}\right)$ (resp. $a \in\left(a^{*}, \infty\right)$ ), the solution $u(., a)$ of $(P)$ is nonpositive.

In what remains we are interested to the solutions with a compact support.

Proposition 2.5. Assume $p>2,0<\alpha<N \beta,-\frac{p}{p-1}<l<0$ and $-N<l<0$ and $q \neq 1-\frac{l(p-2)}{p}$. Then, there exists a constant $a_{0}>0$ such that $u\left(., a_{0}\right)$ has a finite support.

Proof. First we claim that the set

$$
A_{+}=\{a>0: u(r, a)>0, \text { for any } r \geqslant 0\}
$$

is open. In fact, in view of Proposition 1.2, the set $A_{+}$is not empty. In order to prove that $A_{+}$is an open set, take $a_{0} \in A_{+}$. There exists a large $r_{0}$ such that $J\left(r_{0}, a_{0}\right)>0$ where the function $J$ is given by

$$
J(r)=J(r, a)=\left[u(r, a)+\frac{1}{\beta r}\left|u^{\prime}\right|^{p-2} u^{\prime}(r, a)\right] r^{N}
$$

and also we have $J^{\prime}\left(r_{0}, a_{0}\right)>0$. By local continuous dependence of the solutions on the initial value, there is a neighbourhood $O\left(a_{0}\right)$ of $a_{0}$ such that the following holds for any $a \in O\left(a_{0}\right)$

$$
\begin{cases}u(r, a)>0 & \text { in }\left[0, r_{0}\right] \\ J\left(r_{0}, a\right)>0 & \text { and } J^{\prime}\left(r_{0}, a\right)>0\end{cases}
$$

We assert that $J(r, a)$ and $J^{\prime}(r, a)$ are strictly positive for any $r \geqslant r_{0}$ and $a \in O\left(a_{0}\right)$. Otherwise, there exists $r_{1}>r_{0}$ such that $J^{\prime}\left(r_{1}, a\right)=0\left(r_{1}\right.$ is the first zero of $\left.J^{\prime}\left(r_{1}, a\right)\right)$. Then, $J^{\prime}(r, a)>0$ for all $r \in\left[r_{0}, r_{1}[\right.$. Since

$$
J^{\prime}(r)=\frac{1}{\beta} r^{N-1}\left[N \beta-\alpha-r^{l} u^{q-1}\right] u .
$$


the function $g$ given by

$$
g(r)=N \beta-\alpha-r^{l} u^{q-1}
$$

is strictly positive in $\left[r_{0}, r_{1}\left[\right.\right.$. Moreover, as $g^{\prime}(r, a)$ is given by

$$
g^{\prime}(r, a)=r^{l-1}\left[-l u(r, a)-(q-1) r u^{\prime}(r, a)\right] u^{q-2}(r, a) .
$$

We deduce that $g^{\prime}(r, a)>0$ for all $r \in\left[r_{0}, r_{1}\left[\right.\right.$. On the other hand $J^{\prime}\left(r_{1}, a\right)=$ 0 is equivalent to $\frac{1}{\beta} r_{1}^{N-1} g\left(r_{1}, a\right) u\left(r_{1}, a\right)=0$. Then, two cases come; either $u\left(r_{1}, a\right)=0$ or $g\left(r_{1}, a\right)=0$. If $u\left(r_{1}, a\right)=0$, then

$$
J\left(r_{1}, a\right)=\frac{r^{N-1}}{\beta}\left|u^{\prime}\right|^{p-2} u^{\prime}\left(r_{1}, a\right) \leq 0 .
$$

But on the left of $r_{1}$, the function $J$ is strictly positive and strictly increasing, which is impossible. In second case where $g\left(r_{1}, a\right)=0$, we have also a contradiction, because $g(r, a)>0$ and $g^{\prime}(r, a)>0$ for all $r$ in $\left[r_{0}, r_{1}[\right.$. Then,

$$
J(r, a)>0 \text { and } J^{\prime}(r, a)>0
$$

for all $r \in\left[r_{0},+\infty\left[\right.\right.$ and all $a \in O\left(a_{0}\right)$. In particular $u(r, a)>0$ in $\left[r_{0},+\infty[\right.$. Combining this, with (2.37) we deduce that $A_{+}$is open.

To finish the proof, we set $A_{-}=\{a>0: u(r, a)$ is nonpositive $\}$.

Note that $A_{-}$is obviously open and nonempty (from Corollary 2.1).

Since $A_{+}$and $A_{-}$are nonempty and open, the complementary of $A_{+} \cup A_{-}$ is nonempty. This implies that there exists an initial value $a>0$ such that $u(r, a)$ has a compact support.

The proof is complete.

We can easily show the following behavior of the solutions which have compact support

Proposition 2.6. Assume $p>2,0<\alpha<N \beta,-\frac{p}{p-1}<l<0$ and $-N<l$. Let $u$ be a solution with a compact support $[0, R]$. Then

$$
\lim _{r \rightarrow R} \frac{\left|u^{\prime}\right|^{p-1}}{u}(r)=\beta R
$$

We finish this section with the following result

Proposition 2.7. Assume $0<\alpha \leq N \beta,-p<l<0$ and $-N<l$. Then for each $a>0$ the solution $u(r, a)$ has the same sign for large $r$. 
Proof. Let $a>0$ and assume that $u(r, a)$ changes its sign for large $r$. Let $r_{0}$ and $r_{1}$ be two consecutive large zeroes. Integrating the equation

$$
(P)\left\{\begin{array}{l}
\left(\left|u^{\prime}\right|^{p-2} u^{\prime}\right)^{\prime}+\frac{N-1}{r}\left|u^{\prime}\right|^{p-2} u^{\prime}+\alpha u+\beta r u^{\prime}+r^{l}|u|^{q-1} u=0 \\
u(0)=a
\end{array}\right.
$$

on $\left(r_{0}, r_{1}\right)$, we get

$$
\begin{aligned}
r_{1}^{N-1}\left|u^{\prime}\right|^{p-2} u^{\prime}\left(r_{1}\right)- & r_{0}^{N-1}\left|u^{\prime}\right|^{p-2} u^{\prime}\left(r_{0}\right) \\
= & -\int_{r_{0}}^{r_{1}} r^{N-1} u(r)\left[\alpha-N \beta+r^{l}|u|^{q-1} u(r)\right] d s
\end{aligned}
$$

Assume that $u$ is strictly positive on $\left(r_{0}, r_{1}\right)$ (the other case is proved in the same way). Then, the left hand side of (2.41) is strictly negative. On the other hand, invoking Proposition 3.1 and using $0<\alpha \leq N \beta$, we deduce that the right hand side is positive. This is a contradiction, and the proof is complete.

\section{References}

[1] H. Amann, Ordinary Differential Equations, Walter de Gruyter Inc., Germany, 1996.

[2] B. Hamri, A. Gmira, Y. Jabri, Existence and uniqueness of selfsimilar solutions of nonhomogeneous equation, To Appear.

[3] B. Hamri, A. Gmira, Y. Jabri, Asymptotic behavior of selfsimilar solutions of Nonhomogeneous equation, To Appear.

[4] B. Benyounes, A. Gmira, On the radial solutions of degenerate quasilinear elliptic equation, Annales de la Faculté des Sciences de Toulouse, 8, No. 3 (1999), 411-438.

[5] M.F. Bidaut-Véron, S. Pohozaev, Nonexistence results and estimates for some nonlinear elliptic problems, J. Anal. Math., 84 (2001), 1-49.

[6] S. Filippas, A. Tertikas, On similarity solutions of a heat equation with a nonhomogeneous nonlilearity, J. Diff. Equ., 165 (2000), 468-492.

[7] A. Haraux, F.B. Weissler, Non-uniqueness for a semilinear initial value problem, Indiana Univ. Math. J., 31 (1982), 167-189. 
[8] M. Hirose, E. Yanagida, Global structure of self-similar solutions for semilinear heat equations, J. Diff. Equ., 163 (2000), 407-428.

[9] L.A. Peletier, D. Terman, F.B. Weissler, On the equation $\triangle u+\frac{1}{2} x . \nabla u+$ $f(u)=0$, Archive for Rational Mechanics and Analysis, 94 (1986), 83-99.

[10] Y. Naito, T. Suzuki, Radial symmetry self- similar solutions for semilinear heat equations, J. Diff. Equ., 163 (2000), 407-428.

[11] Y.W. Qi, the global existence and non uniqueness of a non linear degenerate equation, Nonlinear Analysis: Theory, Methods and Applications, 31, No. 9 (1998), 117-136.

[12] E. Yanagida, Uniqueness of rapidly decaying solutions to the Haraux weissler equation, J. Diff. Equ., 127 (1996), 561-570. 
